\title{
Relación entre cultura digital y aprendizaje autónomo en estudiantes de estudios generales de una universidad privada de Lima
}

\author{
Elizabeth Cosi Cruz $^{1}$, Carlos Alberto Peña Miranda ${ }^{2}$ y Melanio Sempertegui Gonzales ${ }^{3}$
}

Resumen: La crisis sanitaria generada por el COVID-19 ha cambiado todos nuestros modos de vida, entre ellos los que están ligados a la educación pasándose de una enseñanza presencia a una virtual y que ha puesto en evidencia la necesidad de avanzar significativamente en la incorporación de las nuevas tecnologías en el proceso educativo. En este escenario, la presente investigación tuvo como propósito determinar las correlaciones entre la cultura digital y el aprendizaje autónomo en estudiantes de los primeros años de una universidad privada de Lima durante el año 2020. Para el efecto, se hizo uso de un diseño de investigación no experimental de alcance correlacional. La muestra de estudio fue probabilística y estuvo compuesta por un total de 352 estudiantes, a quienes se les administró dos instrumentos de evaluación: el inventario de cultura digital de Marina Kriscautzky y el cuestionario de aprendizaje autónomo de Mercedes López-Aguado. Los resultados estadísticos alcanzados muestran la existencia de correlaciones significativas y positivas entre ambas variables $(\mathrm{r}=0,83)$, así como también se encontraron correlaciones significativas entre las diversas dimensiones de la cultura digital y las diversas dimensiones del aprendizaje autónomo.

Palabras clave: cultura digital; aprendizaje autónomo; pensamiento crítico.

\section{Relationship between digital culture and autonomous learning in general studies students of a Private University of Lima}

\begin{abstract}
The health crisis generated by COVID-19 has changed all our ways of life, including those that are linked to education, going from a teaching presence to a virtual one and that has highlighted the need to advance significantly in the incorporation of the new technologies in the educational process. In this scenario, the present research aimed to determine the correlations between digital culture and autonomous learning in students of the first years of a private university in Lima during 2020. For this purpose, a research design was used non-experimental of correlational scope. The study sample was probabilistic and consisted of a total of 352 students, who were administered two assessment instruments: Marina Kriscautzky's digital culture inventory and Mercedes López-Aguado's autonomous learning questionnaire. The statistical results achieved show the existence of significant and positive correlations between both variables $(\mathrm{r}=0,83)$, as well as significant correlations between the various dimensions of digital culture and the various dimensions of autonomous learning.
\end{abstract}

Keywords: digital culture; autonomous learning; critical thinking.

Recibido: 10/07/2020. Aceptado: 18/11/2020. Publicado online: 31/12/2020.

\footnotetext{
(CLos autores. Este artículo es publicado por la Revista PESQUIMAT de la Facultad de Ciencias Matemáticas, Universidad Nacional Mayor de San Marcos. Este es un artículo de acceso abierto, distribuido bajo los términos de la licencia Creative Commons Atribucion-No Comercia-Compartir Igual 4.0 Internacional.(http://creativecommons.org/licenses/by-nc-sa/4.0/) que permite el uso no comercial, distribución y reproducción en cualquier medio, siempre que la obra original sea debidamente citada. Para información, por favor póngase en contacto con revistapesquimat.matematica@unmsm.edu.pe

${ }^{1}$ Universidad Privada Norbert Wiener, e-mail: elizabeth.cosi@uwiener.edu.pe

${ }^{2}$ UNMSM, Facultad de Ciencias Matemáticas, e-mail: cpenam@unmsm.edu.pe

${ }^{3}$ UNMSM, Facultad de Ciencias Matemáticas, msempertegui@unmsm.edu.pe
} 


\section{Introducción}

En el inicio del siglo de lo digital, más de una tercera parte de la población mundial se encuentran incorporadas al sistema digital: sus alcances y sus trascendencias que le van dando un nuevo rumbo a los habitantes del planeta en términos de costumbres, hábitos, estilos de vida y comportamiento, generándose una nueva cultura: la cultura digital.

Para Roca (2012), existen dos tipos de tecnologías que han marcado el rumbo de la humanidad a través de los tiempos: por un lado tenemos a las tecnologías del sistema productivo, vinculadas a la fabricación de instrumentos, la domesticación de los animales, la agricultura, la construcción de viviendas hasta llegar a la tecnología digital que ha cambiado los modelos de vida, la economía, el trabajo, etc. De otro lado se tiene a las tecnologías vinculadas a la transmisión de la información y el conocimiento, como son la imprenta, la más media y el Internet.

Desde estos puntos de vista Roca (2012), considera que por primera vez en el escenario mundial se presenta una tecnología sobre la que pueden converger las dos tecnologías señaladas anteriormente, es decir "las tecnologías productivas con las tecnologías de la información y el conocimiento", esto es la tecnología digital. El surgimiento y evolución de esta tecnología digital, ha transformado sensiblemente todo el sistema de relaciones establecidos por la humanidad, por lo que se ven afectados lo social, cultural y lo educativo, pero también la economía, la cultura, los estilos de vida, etc. En síntesis, todo lo vinculado al desarrollo humano, se ha visto afectado.

Sin embargo y a pesar de la magnitud lograda por el avance de las TIC, se ha presentado un fenómeno que se ha hecho evidente y es el de la exclusión tecnológica. Desde los años noventa, sobre todo en los países de menor desarrollo, se ha formulado el concepto de "brecha digital", con el cual se conoce a las desigualdades que, en términos de desarrollo tecnológico, se presenta entre los países y aun en las diferentes regiones en las que se divide un mismo país.

Regularmente "los indicadores que se utilizaban para medir la brecha digital" estaban referidas a la infraestructura tecnológica instalada, y su utilización en los sistemas de producción y de servicios. En épocas más recientes la noción de brecha digital se ha ampliado hasta abarcar campos como la educación, la cultura, lo social, etc., es decir que estos aspectos van a ser importantes a la hora de determinar si se está o no, incluido digitalmente, con lo que las brechas se han ampliado grandemente.

En el terreno de la educación, como se ha señalado, las brechas digitales son bastante grandes y se aprecian tanto en las ciudades como en las zonas rurales, sobre todo en tiempos como los actuales, en las que la educación virtual se ha transformado en una necesidad por los efectos del COVID-19 y la cuarentena obligatoria que ha impuesto el gobierno para combatir este mal. Muchos docentes han tenido y tienen que aprender un conjunto de recursos digitales para poder enfrentar los nuevos desafíos y los estudiantes, mejor aprovisionados en términos de cultura digital, esperan que la calidad educativa no solo no se vea afectada negativamente, sino que mejore sustantivamente.

Desde las propuestas formuladas por Bonder (2008:1), se estima que el desarrollo y masiva utilización de las TIC, se ha producido una fuerte asociación entre la juventud y las tecnologías digitales (llámese dispositivos móviles, Internet y computadora), por lo que algunos autores como Prensky (2013), han llamado a los niños y adolescentes, "nativos digitales" o "e-generación" o simplemente "millennials", quienes tienen características peculiares que difieren de las generaciones anteriores por lo que sus hábitos culturales han sufrido cambios significativos. La cultura digital es parte del mundo de las nuevas generaciones pero sin embargo, la educación no ha 
marchado al ritmo de los avances de las tecnologías digitales ni de los cambios que se han ido produciendo en la vida de los niños y jóvenes.

Prensky (2013), insiste mucho en que los estudiantes están cambiando, a partir de las nuevas experiencias que se están generado por la utilización de la tecnología digital fuera de los centros educativos, básicos o superiores, y que, por tanto, estas instituciones los alejan del mundo en el que se desenvuelven regularmente.

A estas condiciones se enfrentan los docentes de hoy y en este contexto tienen que desarrollar sus clases con la utilización de las herramientas digitales de manera que los estudiantes puedan producir "conocimiento relevante, sean autónomos, discriminen fuentes confiables y trabajen colaborativamente". La autonomía del aprendizaje es una de las tareas esenciales en la educación de hoy y se espera que con la utilización de la tecnología digital que los estudiantes tan bien utilizan, se logre que el aprendizaje conectado con la realidad, se vuelva atractivo y útil para su futuro.

La importancia que tiene para el país, el hecho de que los estudiantes puedan estar en condiciones de establecer sus propios aprendizajes, se va a expresar en una suerte de desarrollo intelectual que de manera colectiva se va formulando desde el propio estudiante puesto que son sus conocimientos los que le permitirán enfrentar y entender las diversas situaciones que se le presenten en la vida diaria. A partir de ello será capaz de explicarlas utilizando sus propios conceptos, produciéndose la gran trasformación pues pasara de ser un simple "espectador" para convertirse en "actor" puesto que será capaz de formular soluciones novedosas a los problemas existentes y que estén por venir.

Bajo estas consideraciones se debe entender que el aprendizaje autónomo es una de las competencias fundamentales para lograr el éxito académico y formativo de los estudiantes universitarios, por lo que deviene en un elemento de gran importancia que se debe considerar en el sistema universitario.

Por estas razones es que las universidades deben procurar la contratación de personal docente, que no solo dispongan de los conocimientos necesarios en su materia, sino que además y de manera prioritaria, estén muy bien informados sobre las nuevas tendencias pedagógicas y didácticas que rigen la educación superior de manera que se pueda fortalecer las competencias de los estudiantes y que les permitan desarrollar con total eficacia las tareas propias de su profesión.

El objetivo fundamental del trabajo de investigación, está dirigido al establecimiento de relaciones entre la cultura digital y el aprendizaje autónomo en una muestra de estudiantes de estudios generales de una universidad privada de Lima.

\section{Metodología}

En la presente investigación se empleó el método hipotético-deductivo, el mismo que es conceptualizado por Bernal (2010), como "un procedimiento que parte de unas aseveraciones en calidad de hipótesis y busca refutar o falsear tales hipótesis, deduciendo de ellas conclusiones que deben confrontarse con los hechos"(p. 56), tal como se ha hecho a lo largo de la presente investigación, particularmente en lo que se refiere a los resultados alcanzados.

\section{Diseño de investigación}

Por las necesidades de la investigación, el diseño fue no experimental de alcance correlacional y transversal, siguiendo lo propuesto por Hernández, Fernández y Baptista (2014). En términos 
específicos este diseño busca determinar si dos o más variables se vinculan entre si en un momento en el tiempo. "Las variables se interrelacionan bajo el siguiente esquema:"

$\mathrm{Su}$ diagrama representativo es el siguiente:

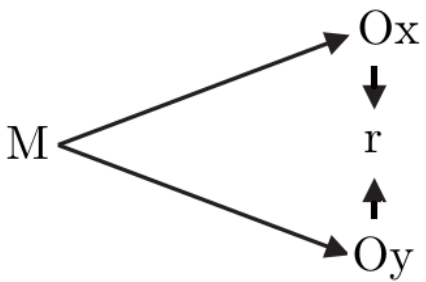

En el esquema:

"M = Representa la muestra de estudio

$\mathrm{Ox}=$ Representa los datos de la variable cultura digital

Oy $=$ Representa los datos de la variable aprendizaje autónomo

$\mathrm{r}=$ Indica el grado de correlación entre ambas variables"

\section{Muestra}

"El diseño de la muestra fue probabilístico y de tipo estratificado", distribuyendo a los estudiantes según la carrera profesional de procedencia. La estimación de la muestra se realizó de acuerdo al tamaño poblacional que, en el presente caso, fue de 4166 estudiantes. De este total "se tomó una muestra representativa mediante la técnica de muestreo aleatorio y por afijación proporcional" (Hernández et. al, 2014, p.16), la misma que dio como resultado que la muestra estuviera compuesta por 352 estudiantes.

Tabla 1. Composición de la muestra por ciclo académico

\begin{tabular}{|c|c|c|}
\hline Ciclo & Frecuencia & Porcentaje \\
\hline I & 105 & 29,8 \\
\hline II & 90 & 25,6 \\
\hline III & 69 & 19,6 \\
\hline IV & 88 & 25,0 \\
\hline Total & 352 & 100,0 \\
\hline
\end{tabular}

\section{Instrumentos}

Los instrumentos empleados en la investigación fueron el Inventario de cultura digital de Marina Kriscautzky de la Universidad Nacional Autónoma de México (UNAM) y el Cuestionario de aprendizaje autónomo de Mercedes López-Aguado de la Universidad de León, España.

\section{Análisis de validez y confiabilidad de los instrumentos}

Los dos instrumentos que se utilizaron en el presente trabajo de investigación fueron sometidos a los análisis estadísticos respectivos, a fin de determinar si presentaban consistentes niveles de validez y confiabilidad que garantizaran la justeza de los datos a obtener y que servirían para determinar los resultados que probarían las hipótesis planteadas. Los análisis estadísticos efectuados arrojaron como resultado que, el análisis de confiabilidad realizado con el estadístico 
alfa de Cronbach, el cuestionario de cultura digital alcanza un valor de 0,94 el mismo que puede ser tipificado como muy bueno. En el caso del cuestionario de aprendizaje autónomo, el valor alcanzado fue de 0,89, el cual es igualmente bueno. Para el caso de la "validez de los instrumentos, estos se realizaron utilizando el análisis factorial exploratorio". Los resultados encontrados indican que ambas pruebas presentan validez de Constructo.

\section{Resultados}

\section{Análisis descriptivos}

El resultado del análisis de normalidad efectuado con "el test de bondad de ajuste a la curva normal de Kolmogorov-Smirnov" y que se observan en la tabla 2, muestran que no "existe una adecuada aproximación a la curva normal en tanto los coeficientes K-S Z obtenidos son estadísticamente significativos, por lo que en el presente caso se deben utilizar para el análisis de los datos, estadísticas no paramétricas"(Siegel y Castellan, 1995, p. 20).

Tabla 2. Análisis de normalidad de las variables estudiadas

\begin{tabular}{|l|c|c|c|c|}
\hline Variable & Media & D. E. & K-SZ & Sig. \\
\hline Búsqueda de información & 29,60 & 5,05 & 0,104 & 0,000 \\
\hline Conocimientos y Uso de las TIC & 34,61 & 5,05 & 0,106 & 0,00 \\
\hline Organizar y crear contenidos digit & 18,19 & 3,33 & 0,118 & 0,000 \\
\hline Ética y Seguridad & 27,47 & 3,67 & 0,157 & 0,000 \\
\hline Resolución de problemas & 23,08 & 3,12 & 0,145 & 0,000 \\
\hline & & & & \\
\hline Estrategias de Ampliación & 35,69 & 2,49 & 0,135 & 0,000 \\
\hline Estrategias de Colaboración & 39,30 & 2,43 & 0,095 & 0,000 \\
\hline Estrategias de Conceptualización & 30,56 & 2,43 & 0,114 & 0,000 \\
\hline Estrategias de Planificación & 19,38 & 2,58 & 0,168 & 0,000 \\
\hline Estrategias de Preparación de exám & 24,02 & 3,02 & 0,149 & 0,000 \\
\hline Estrategias de Participación & 23,76 & 3,02 & 0,136 & 0,000 \\
\hline
\end{tabular}

$N=352$

Los resultados del análisis descriptivo que se presentan en la tabla 3 y figura 1 , indican que la cultura digital prevalentemente se encuentra ubicada en un nivel medio $63,9 \%$, mientras que el $18,2 \%$ se encuentran ubicados a nivel alto y $17,9 \%$ en el nivel bajo.

Tabla 3. Niveles de cultura digital

\begin{tabular}{|c|c|c|}
\hline Niveles & Frecuencias & Porcentajes \\
\hline Bajo & 63 & 17,9 \\
\hline Medio & 225 & 63,9 \\
\hline Alto & 64 & 18,2 \\
\hline Total & 352 & 100,0 \\
\hline
\end{tabular}



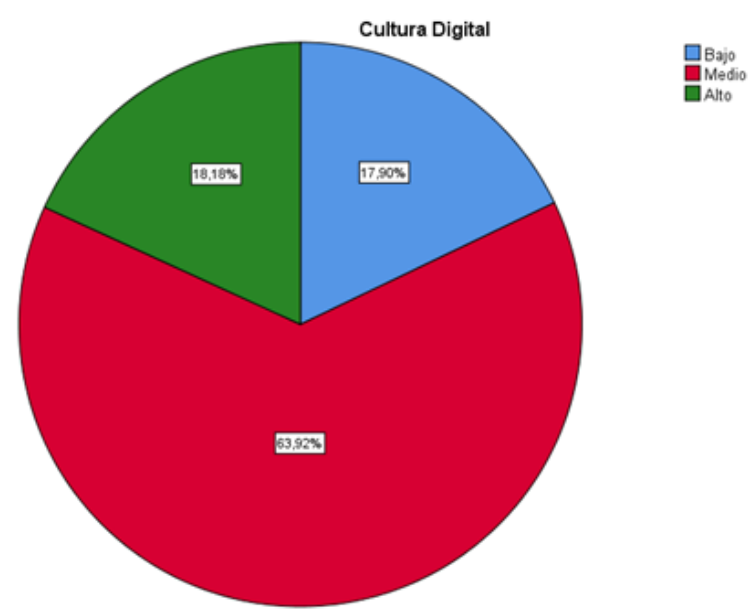

Figura 1. Niveles de cultura digital

Del análisis descriptivo que se observan en la tabla 4 y figura 2 , indica que el aprendizaje aut ónomo prevalentemente se encuentra ubicada a nivel medio con el 64,8 \%, mientras que el 18,8\% se ubica a un nivel bajo y el 16,5\% se encuentran en el nivel alto.

Tabla 4. Niveles de aprendizaje autónomo

\begin{tabular}{|c|c|c|}
\hline Niveles & Frecuencias & Porcentajes \\
\hline Bajo & 66 & 18,8 \\
\hline Medio & 228 & 64,8 \\
\hline Alto & 58 & 16,5 \\
\hline Total & 352 & 100,0 \\
\hline
\end{tabular}

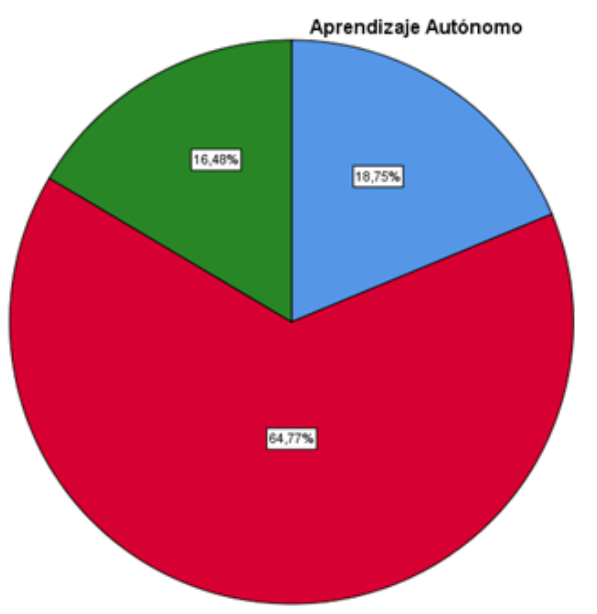

Figura 2. Niveles de aprendizaje autónomo 


\section{Análisis correlacionales descriptivos}

El análisis de las correlaciones efectuadas con el "estadístico rho de Spearman" que se presentan en la Tabla 5 y figura 3, indican que la cultura digital se correlaciona de manera significativa con el aprendizaje autónomo en la muestra estudiada.

Tabla 5. Análisis de correlación (rho de Spearman) entre las diversas dimensiones de la cultura digital y el aprendizaje autónomo

\begin{tabular}{|c|c|}
\hline Variables & Aprendizaje autónomo \\
\hline Cultura digital & $0,83^{* * *}$ \\
\hline
\end{tabular}

${ }^{*} p<0,05^{* *} p<0,01^{* * *} p<0,001$

$N=352$

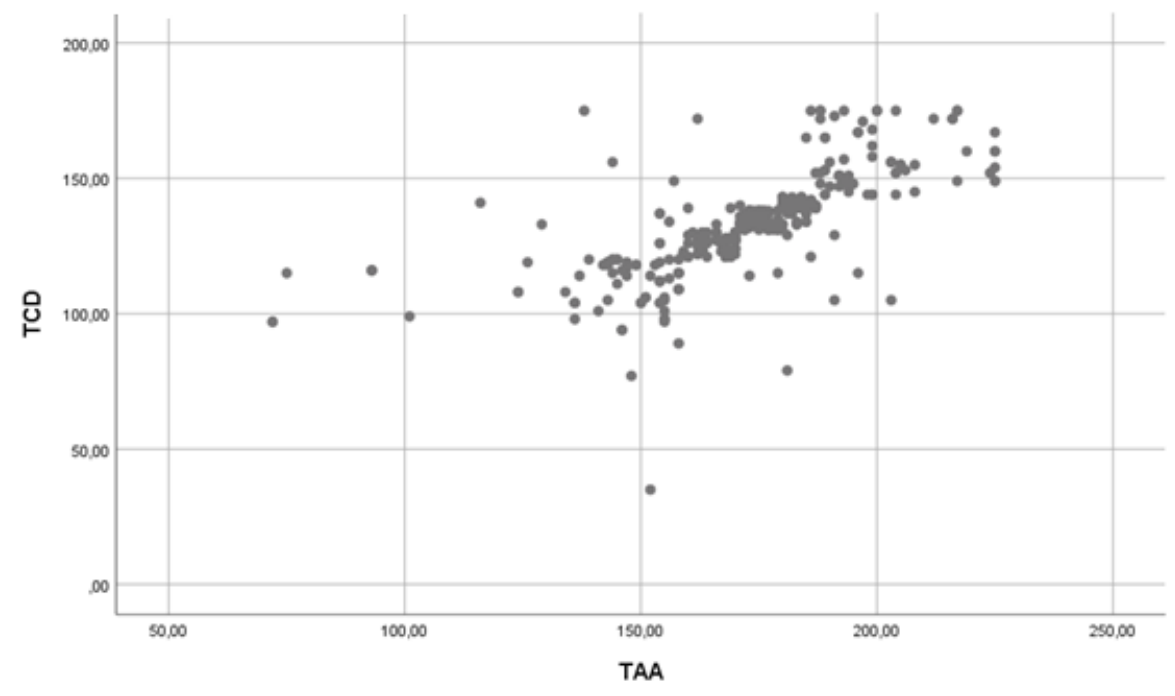

Figura 3. Correlación entre la cultura digital y el aprendizaje autónomo

En un intento por complementar los resultados anteriormente expuestos se realizó el análisis de tabulación cruzada de acuerdo a los niveles que presentaban la cultura digital y el aprendizaje autónomo, los resultados mostrados en la tabla 6, indican que existen diferencias estadísticas significativas en la distribución de las celdas (Chi-cuadrada $=388,27 \mathrm{p}>0,001$ ), adicionalmente el coeficiente gamma indica que las variables se encuentran relacionadas significativamente $($ Gamma $=0,897 \mathrm{p}<0,001)$. 
Tabla 6. Análisis de tabulación cruzada entre la cultura digital y el aprendizaje autónomo

\begin{tabular}{|c|c|c|c|c|c|}
\hline & \multicolumn{3}{|c|}{ Aprendizaje Autónomo } & \multirow{2}{*}{ Total } \\
\hline & & Bajo & Medio & Alto & \\
\hline \multirow{3}{*}{$\begin{array}{l}\text { Cultura } \\
\text { Digital }\end{array}$} & Bajo & 51 & 9 & 3 & 63 \\
\hline & Medio & 13 & 205 & 7 & 225 \\
\hline & Alto & 2 & 14 & 48 & 64 \\
\hline \multicolumn{2}{|c|}{ Total } & 66 & 228 & 58 & 352 \\
\hline \multicolumn{6}{|c|}{ Chi-cuadrado de Pearson $=388,27$ Gl. $=4$ sig. $=0,00$} \\
\hline \multicolumn{6}{|c|}{ Gamma $=0,897$ sig $=0,001$} \\
\hline
\end{tabular}

${ }^{*} p<0,05^{* *} p<0,01^{* * *} p<0,001$

$N=352$

Los resultados del análisis correlacional que se aprecian en la Tabla 7, nos informan que las diversas dimensiones de la cultura digital se correlacionan "significativamente con el aprendizaje autónomo de los estudiantes".

Tabla 7. Análisis de correlación (rho de Spearman) entre las diversas dimensiones de la cultura digital y el aprendizaje autónomo

\begin{tabular}{|c|c|}
\hline Variables & Aprendizaje autónomo \\
\hline Búsqueda de información & $0,78^{* * *}$ \\
\hline Conocimientos y Uso de las TIC & $0,73^{* * *}$ \\
\hline Organizar y crear contenidos digitales & $0,70^{* * *}$ \\
\hline Ética y Seguridad & $0,69^{* * *}$ \\
\hline Resolución de problemas & $0,68^{* * *}$ \\
\hline
\end{tabular}

${ }^{*} p<0,05^{* *} p<0,01^{* * *} p<0,001$

$N=352$

Los resultados del análisis correlacional que se aprecian en la Tabla 8, nos informan que las diversas dimensiones de la cultura digital se "correlacionan significativamente con las diversas dimensiones del aprendizaje autónomo de los estudiantes".

Tabla 8. Análisis de las correlaciones entre las diversas áreas de las competencias digitales y las diversas habilidades investigativas

\begin{tabular}{|c|c|c|c|c|c|c|}
\hline Variables & EAmp & ECol & ECon & EPla & EPre & EPar \\
\hline Búsqueda de información & $0,61^{* *}$ & $0,72^{* *}$ & $0,58^{* *}$ & $0,61^{* *}$ & $0,58^{* *}$ & $0,63^{* *}$ \\
\hline Conocimientos y Uso de las TIC & $0,54^{* *}$ & $0,62^{* *}$ & $0,61^{* *}$ & $0,58^{* *}$ & $0,59^{* *}$ & $0,60^{* *}$ \\
\hline Organizar y crear contenidos digitales & $0,53^{* *}$ & $0,62^{* *}$ & $0,57^{* *}$ & $0,52^{* *}$ & $0,56^{* *}$ & $0,58^{* *}$ \\
\hline Ética y Seguridad & $0,51^{* *}$ & $0,59^{* *}$ & $0,59^{* *}$ & $0,58^{* *}$ & $0,55^{* *}$ & $0,62^{* *}$ \\
\hline Resolución de problemas & $0,52^{* *}$ & $0,58^{* *}$ & $0,53^{* *}$ & $0,54^{* *}$ & $0,58^{* *}$ & $0,55^{* *}$ \\
\hline
\end{tabular}

${ }^{*} p<0,05^{* *} p<0,01^{* * *} p<0,001$

$N=352$

EAmp $=$ Estrategias de Ampliación, ECol = Estrategias de Colaboración, ECon $=$ Estrategias de Conceptualización, EPla = Estrategias de Planificación, EPre = Estrategias de Preparación de exámenes, EPar $=$ Estrategias de Participación 


\section{Discusión de los resultados}

En el desarrollo del presente trabajo de investigación se debió hacer uso de los recursos tecnológicos disponibles, a fin de recolectar los datos de la muestra de manera virtual, en la medida de que no es posible hacerlo de manera presencial. Una ventaja que se tuvo es que, al ser la muestra, estudiantes de la universidad fue relativamente sencillo que accedieran a responder las encuestas que se les envío a través de sus correos electrónicos.

Los resultados que se obtuvieron en la presente investigación informan que, respecto de la primera hipótesis que se formuló; "La cultura digital se relaciona significativamente con el aprendizaje autónomo en estudiantes de estudios generales de una universidad privada de Lima", que la misma ha sido respaldada, en tanto el análisis de correlación alcanza un valor de $\mathrm{r}=0,83 \mathrm{el}$ que se puede considerar como alto. Estos resultados refuerzan las propuestas de Prensky (2013), en el sentido de que los estudiantes que han nacido en este siglo XXI y a los que llama nativos digitales, son personas que conocen y manejan fácilmente las herramientas tecnológicas, tienen un acceso rápido y fácil a la información. Se movilizan en estos entornos, conocen las redes sociales, y están en constante información de la dinámica de estas herramientas por lo que sus procesos de aprendizaje se facilitan cuando deben hacerlo apoyados en las TICs. Sin embargo y siguiendo a Gimeno Sacristán, (2013), la presencia de las TIC en nuestras vidas ha significado cambios dramáticos en nuestras formas de vida y también, por supuesto, en las prácticas educativas por lo que su presencia en ellas debe adscribirse a un proyecto transformador de todo el proceso de enseñanza aprendizaje que implique cambios de orden pedagógico y didáctico de manera que se pueda adecuar a los cambios tecnológicos.

Estos resultados nos dejan claramente establecido que la cultura digital es una necesidad urgente de atender y desarrollar en los momentos actuales en la medida de que todo gira en torno a las nuevas TIC y porque los procesos educativos se ven facilitados en los jóvenes estudiantes en tanto son ellos lo que mejor conocen y se adaptan a estas tecnologías. En esta línea Pérez (2015), considera que es tiempo de preparar y capacitar a docentes y estudiantes en el manejo de las nuevas tecnologías, en tanto deben convertirse en las "capacidades operativas, instrumentales y actitudinales con las que funcionar y resolver problemas en la era digital y, sin cuyo dominio, los alumnos estarán perdidos y serán analfabetos funcionales"(p. 573).

\section{Conclusiones}

1. La cultura digital mostrada en los resultados de la presente investigación, indica que la misma se ubica fundamentalmente en un nivel medio.

2. El aprendizaje autónomo mostrado en los resultados de la presente investigación, indica que la misma se ubica fundamentalmente en un nivel medio.

3. El análisis de las correlaciónales que se realizaron con el apoyo del estadístico rho de Spearman y el análisis de tabulación cruzada y el estadístico gamma, nos indican la existencia de correlaciones significativas entre la cultura digital y el aprendizaje autónomo, en los estudiantes de la muestra.

4. El análisis de las correlaciónales que se realizaron con el apoyo del estadístico rho de Spearman, nos indican la existencia de "correlaciones significativas entre las diversas dimensiones" de la cultura digital y el aprendizaje autónomo, en los estudiantes de la muestra.

5. El análisis de las correlaciónales que se realizaron con el apoyo del estadístico rho de Spearman, nos indican la existencia de "correlaciones significativas entre las diversas di- 
mensiones" de la cultura digital y las diversas dimensiones del aprendizaje autónomo, en los estudiantes de la muestra.

\section{Referencias bibliográficas}

[1] Bonder, G. (2008). Juventud, género y TIC: Imaginarios en la construcción de la Sociedad de la Información en América Latina. En: ARBOR CLXXXIV 733 septiembre-octubre, 917-934. Recuperado el 10 de julio de 2011 de: http://arbor.revistas.csic.es/index.php/arbor/issue/view/ 17/showToc

[2] Gimeno Sacristán, J. (2013). En busca del sentido de la educación. Madrid: Ediciones Morata.

[3] Hernández, R.; Fernández, C. y Baptista, P. (2014). Metodología de la Investigación. México: Ed Mc Graw Hill.

[4] Herrera, A. (2015). Una mirada reflexiva sobre las TIC en Educación Superior. Revista Electrónica de Investigación Educativa. 17 (1) 1-4.

[5] Martín, E. y Moreno, A. (2009). Competencia para aprender a aprender. Madrid: Alianza Editorial.

[6] Organización de las Naciones Unidas para la Educación, la Ciencia y la Cultura (Unesco) (2008). Estándares de Competencias en TIC para Docentes. Recuperado de: http://www. unesco.org/en/competencystandards-teachers

[7] Prensky, M. (2013). Enseñar a nativos digitales (1a. ed). México: SM Ediciones

[8] Sierra Bravo, R. (2003). Tesis doctorales y trabajos de investigación científica. Madrid, España: Ediciones Paraninfo S.A. 